\title{
Civilisations
}

Revue internationale d'anthropologie et de sciences

humaines

39 | 1991

Japon : les enjeux du futur

\section{Le changement technique et l'acquisition de nouvelles compétences dans les PMI japonaises}

\section{Yveline Lecler}

\section{(2) OpenEdition \\ Journals}

Édition électronique

URL : http://journals.openedition.org/civilisations/1660

DOI : 10.4000/civilisations. 1660

ISSN : 2032-0442

\section{Éditeur}

Institut de sociologie de l'Université Libre de Bruxelles

\section{Édition imprimée}

Date de publication : 30 octobre 1991

Pagination : 249-297

ISBN : 2-87263-044-9

ISSN : 0009-8140

Référence électronique

Yveline Lecler, « Le changement technique et l'acquisition de nouvelles compétences dans les PMI japonaises », Civilisations [En ligne], 39 | 1991, mis en ligne le 06 juillet 2009, consulté le 19 avril 2019. URL : http://journals.openedition.org/civilisations/1660 ; DOI : 10.4000/civilisations.1660 


\title{
LE CHANGEMENT TECHNIQUE ET L'ACQUISITION DE NOUVELLES COMPETENCES DANS LES PMI JAPONAISES
}

\author{
Yveline LECLER
}

Introduction

Les années 70 et 80 sont marquées par une accélération du changement technique dans les entreprises japonaises, tant au niveau production qu'au niveau gestion de production. Après le choc pétrolier de 1973, l'environnement étant plus fluctuant et la concurrence exacerbée, les grandes entreprises se sont lancées dans une vague de rationalisations successives, entraînant, par l'importance de la sous-traitance, les PMI dans leur sillage.

D'abord centrées sur la diminution des coûts de production dans l'ensemble du système productif, tout en assurant au produit une qualité croissante, les priorités se sont portées, pour les PMI, sur l'acquisition d'équipements nouveaux : MOCN, $\mathrm{CU}, \mathrm{RI}$... Ceux-ci tout en constituant une réponse aux impératifs de qualité permettaient une flexibilisation indispensable pour faire face à l'extension des systèmes "juste à temps" (JAT). Ces derniers ont en effet pour corollaire un fractionnement des séries qui doivent être reprises sur un temps de plus en plus court. Seuls des équipements rapidement reprogrammables, ou pouvant gérer des séries de pièces différentes, pouvaient répondre aux objectifs du JAT : satisfaire les délais de lviraisons du client tout en éliminant les gaspillages dus à la constitution de stocks.

Cette première vague de modernisation plus ou moins digérée, les PMI sous-traitantes se trouvent confrontées à de nouvelles évolutions dans la nature des transactions interentreprises et dans leur modalité de fonctionnement. Les 
grandes entreprises, toujours à la recherche d'une plus grande efficacité du système productif dont elles constituent le sommet, et ce d'autant plus que la hausse du yen a entamé leur compétitivité, entendent se tourner vers l'économie des coûts de gestion. Les PMI sont alors appelées à fournir des ensembles complets ou même des produits finis, et non plus seulement des pièces, ce qui implique de maîtriser des fonctions nouvelles (assemblage, finition, mais aussi conception, développement). Elles doivent également être en mesure de pratiquer l'analyse de la valeur, de s'insérer dans un réseau informatisé piloté par le donneur d'ordres, de développer, à terme, de nouveaux produits ou nouveaux procédés. Elles doivent enfin être capables de pratiquer effectivement le JAT, y compris au niveau de la production et non pas simplement, comme cela est encore souvent le cas dans les années 80 , au niveau de la livraison. Bref, elles doivent intégrer les principes de gestion globale qui signifie diminution des coûts à tous les niveaux simultanément afin de donner à l'ensemble une cohérence totale garantissant la meilleure efficacité de la chaîne de production ${ }^{1}$.

Parallèlement, les PMI ont également à se mobiliser pour faire face à l'internationalisation croissante des grandes entreprises japonaises. Celles-ci cherchent à résoudre les problèmes de tensions commerciales et à s'adapter à la hausse du yen, phénomène plus récent qui provoque d'ailleurs une accélération du mouvement. Tant qu'il n'était question que de mesure anti-tension commerciale, l'impact de l'internationalisation n'était pas encore très sensible au niveau de l'approvisionnement car les entreprises délocalisées continuaient à se fournir au Japon. Mais avec la hausse du yen, l'internationalisation prend une autre dimension car elle s'inscrit alors dans une stratégie industrielle cohérente d'action sur les prix dont le corollaire est l'approvisionnement auprès de fournisseurs locaux ${ }^{2}$. Il est donc probable que les PMI, du moins certaines d'entre elles, soient de plus en plus concernées par leur 
propre internationalisation, que ce soit dans les pays développés pour suivre leurs donneurs d'ordres, ou dans les pays d'Asie, pour contrer la hausse du yen.

Il est bien évident que la majorité des PMI sous-traitantes n'est pas encore en capacité de répondre à l'ensemble de ces exigences. Toutes n'y parviendront d'ailleurs pas, ce qui devrait amener un repositionnement, sans rapport avec la taille des entreprises, dans la hiérarchisation verticale (keiretsu). Les $\mathrm{PMI}^{3}$ sont généralement conscientes des risques qui pèsent sur leur devenir et prennent diverses mesures pour s'adapter, se restructurer et survivre.

Parmi ces mesures, et conditionnant la réussite de l'ensemble des stratégies mises en oeuvre, se trouve le besoin d'une action sur la qualité de la main-d'oeuvre. Ainsi, il est probable que les sous-traitants de premier rang de demain seront les entreprises qui auront su s'assurer les services d'un personnel technique et de gestion de bon niveau, capable de dialoguer de plus en plus à travers un réseau informatisé national ou international, non seulement avec les services achats, mais aussi avec les services recherche et développement, planification... du donneur d'ordres.

Par quelles actions et selon quelles procédures les PMI peuvent-elles prétendre à cette évolution des hommes, compte tenu du type de main-d'oeuvre dont elles disposent, des possibilités de recrutement qui leur sont ouvertes et de l'effort de formation qu'elles peuvent consentir ? Pour tenter de répondre à cette question, une première partie fera apparaître les caractéristiques de la main-d'oeuvre des PMI en relation avec les besoins de compétences nouvelles, ainsi que les stratégies développées en matière de recrutement; tandis qu'une deuxième partie analysera les procédures de formation mises en oeuvre dans les PMI ou à leur disposition, même si 
elles les utilisent encore peu, qu'il s'agisse de formation proprement dite ou d'autres mécanismes y contribuant.

\section{La main-d'oeuvre des PMI et le besoin de nouvelles compétences}

Le besoin de compétences nouvelles n'est pas spécifique aux PMI. Les grandes entreprises sont également confrontées à l'émergence de fonctions nouvelles en liaison avec le changement technique qui s'opère. Mais le problème de l'inadéquation entre savoir-faire internes et nouveaux besoins est plus prégnant dans les PMI pour diverses raisons. D'abord, les grandes entreprises ont abordé la micro-électronique, la mise en place des JAT, etc... beaucoup plus tôt que les PMI et ont donc déjà une longue expérience en la matière. Elles ont pu petit à petit adapter leurs recrutements à ces nouvelles fonctions qui apparaissent avec l'électronique par exemple. Elles disposent également de plus grandes potentialités internes quant à la formation de la main-d'oeuvre en place. Si elles n'ont pas résolu tous les problèmes de cet ordre, et expriment encore des besoins, c'est d'une part que l'évolution est permanente, et d'autre part que les recrutements étant moins nombreux que par le passé du fait de l'économie de travail réalisée, le renouvellement de la main-d'oeuvre est plus lent; or les travailleurs âgés, considérés en surnombre, sont souvent estimés non adaptables aux nouvelles technologies. Pour comprendre en quoi le problème se pose de façon accrue pour les PMI, il convient de revenir brièvement sur ce qui caractérise la main-d'oeuvre dans cette catégorie d'entreprise.

\subsection{Les salariés des PMI : un prolongement de tendances anciennes ?}

Les PMI, plus tardivement concernées par le changement technique, partent avec, en quelque sorte, un handicap supplémentaire qui tient à la fois à la composition de la maind'oeuvre en place et à leur position défavorisée sur le marché du travail. En effet, les grandes entreprises, dont l'image de 
marque est largement supérieure et qui offrent traditionnellement des conditions et garanties en matière d'emploi et de carrière plus élevées, drainent vers elles la maind'oeuvre la plus appropriée. Les PMI ont alors des difficultés à s'assurer les services de jeunes sortant directement du système éducatif et ayant le niveau scolaire requis, jeunes qui sont aujourd'hui encore la main-d'oeuvre privilégiée par les entreprises même si les choses ont tendance à évoluer dans ce domaine également ${ }^{4}$.

Il serait hors de propos d'entrer dans une comparaison systématique de la composition du salariat des grandes et des petites entreprises. Les évolutions récentes mises en perspectives des tendances anciennes suffiront à poser le problème.

Une étude antérieure ${ }^{5}$ permet de résumer en quelques mots les caractéristiques de la main-d'oeuvre des PMI par rapport à celle des grandes entreprises : plus fort recours à la maind'oeuvre féminine; importance de l'emploi à temps partiel, notamment féminin; âge moyen beaucoup plus élevé et nombreux recrutements de travailleurs dits "en cours de carrière"6.

Un recours plus important a la main-d'oeuvre féminine et a temps partiel

Avec un pourcentage de main-d'oeuvre féminine passant de $37,3 \%$ en 1975 à $41,8 \%$ en 1985, la féminisation est en progression constante dans les PMI japonaises (- de 500 salariés). Dans un même temps, le pourcentage de femmes parmi les salariés des grandes entreprises (+ de 500 salariés) fluctue à la baisse, passant de l'ordre de $25 \%$ à un peu plus de $20 \%$ et accentuant l'écart entre grandes et petites entreprises. Ainsi, tandis que les PMI comptaient 750.000 femmes de plus, les 
GE en perdaient 10.000. Cette évolution qui va tout à fait dans le sens d'un prolongement des tendances antérieures devrait se modeler dans les années à venir si l'on en croit les perspectives d'embauches qu'affichent les firmes interrogées : $76 \%$ des GE contre seulement $39 \%$ des PMI comptent dorénavant recruter davantage de femmes ${ }^{7}$.

En ce qui concerne l'emploi à temps partiel, c'est-à-dire les personnes travaillant moins de 35 heures par semaine, la tendance est un peu différente car la hausse concerne toutes les catégories d'entreprises. Il convient d'abord de rappeler que l'emploi à temps partiel concerne essentiellement les femmes puisqu'elles en représentent $82,2 \%(1987)^{8}$. La part des femmes à temps partiel sur le total des femmes salariées passe de $17,4 \%$ en 1975 à $22 \%$ en 1985 dans les PMI, enregistrant une progression assez régulière. Dans les grandes entreprises, l'évolution est plus fluctuante : de l'ordre de $10 \%$ en 1973, le pourcentage se situe autour de $15 \%$ en 1975, en 1980, puis en 1984, chacun de ces pics étant suivis d'une légère baisse. Globalement sur la période 1975-1985, l'emploi à temps partiel progresse fortement : $68,2 \%$. Les PMI contribuent davantage à cette progression : +1.130 .000 personnes contre +210.000 personnes pour les $\mathrm{GE}^{9}$.

\section{Un âge moyen plus élevé, mais une ancienneté moyenne inférieure}

Le vieillissement de la population japonaise est rapide et l'âge moyen a tendance à s'élever dans toutes les catégories de firmes. Cependant, sur la décennie 75-85, le pourcentage de travailleurs âgés reste plus important dans les petites entreprises que dans les grandes, même si la progression y est légèrement inférieure : 
Tableau 1: Pourcentage de travailleurs âgés par rapport au total salarié, industrie manufacturière (unité $=\%$ )

1978

1985

\begin{tabular}{lll}
\hline PME & 34,1 & 39,7 \\
GE & 20,3 & 26,3 \\
\hline
\end{tabular}

Source : livre blanc sur les PME, 1988, p. 105.

Il est intéressant de noter à ce sujet la différence de raison du vieillissement entre PMI et GE. En effet, alors que dans les grandes entreprises, c'est principalement la réduction des embauches qui cause une augmentation de l'âge moyen, dans les PMI, c'est l'embauche directe de travailleurs âgés qui en explique l'essentiel. En 1985, sur l'ensemble des industries, le poids des PMI en matière de départ de travailleurs âgés est de $65,6 \%$ tandis que leur poids en matière de recrutement est de $77,9 \%$ (industrie manufacturière, respectivement $75,1 \%$ et $84,8 \%$ ). Ce qui signifie qu'il s'opère un certain transfert des grandes entreprises vers les petites, transferts confirmés par les taux de recrutement de travailleurs de plus de 45 ans en cours de carrière : $6,7 \%$ pour les PMI contre $3,0 \%$ pour les GE toutes industries confondues en 1985 (respectivement 5,0\% et 1,4\% dans l'industrie manufacturière). La raison invoquée par la majorité des PMI pour expliquer cette situation est la difficulté qu'elles ont à embaucher des jeunes diplômés sortant du système éducatif. Ceci dit, les recrutements de travailleurs en cours de carrière ne se limitent pas aux tranches d'âge élevé, notamment en ce qui concerne les PMI qui face à leur problème de recrutement à la sortie de l'école, surtout de l'université, 
tentent d'embaucher par cette procédure les travailleurs les plus jeunes possible.

Tableau 2 : Taux de recrutement des travailleurs en cours de carrière par tranche d'âge et taille d'entreprise, 1987 (hommes)

\begin{tabular}{lccc}
\hline entreprises & grandes & moyennes & petites \\
\hline & & & \\
$25-29$ ans & 1,7 & 4,0 & 11,2 \\
$30-34$ ans & 0,6 & 2,3 & 7,9 \\
$35-39$ ans & 0,2 & 1,7 & 6,6 \\
$40-44$ ans & 0,4 & 1,2 & 5,5 \\
$45-49$ ans & 0,3 & 1,4 & 4,8 \\
$50-54$ ans & 0,4 & 1,5 & 4,5 \\
$55-59$ ans & 0,7 & 3,0 & 5,6 \\
+60 ans & 4,8 & 4,1 & 4,2 \\
\hline
\end{tabular}

Source : livre blanc sur le travail 1988, p. 190

N.B. : pourcentage de salariés de moins d'un an d'ancienneté sur total des salariés de la tranche d'âge.

* tranche d'âge particulière du fait de la retraite et du principe de réemploi (voir note 9).

C'est cette importance des recrutements directs de travailleurs âgés, associée à une rotation de la main-d'oeuvre plus forte, qui explique le différentiel d'ancienneté moyenne qui existe entre PMI et GE. Pour une ancienneté quasi équivalente dans les basses tranches d'âge, l'écart se creuse au fur et à mesure que l'âge avance et que l'entreprise est petite, à l'exception bien sûr des plus de 60 ans, catégorie spéciale du fait de la retraite et du principe de rééemploi ${ }^{10}$. 
Tableau 3: Différentiel d'ancienneté moyenne par taille d'entreprise et tranches d'âge (1987, unité = années)

\begin{tabular}{lcc}
\hline entreprises & grandes-moyennes & moyennes-petites \\
\hline - 17 ans & 0,2 & 0,2 \\
$18-19$ ans & 0,1 & 0,2 \\
$20-24$ ans & 0,3 & 0,3 \\
$25-29$ ans & 0,4 & 1,1 \\
$30-34$ ans & 2,1 & 2,8 \\
$35-39$ ans & 2,5 & 4,2 \\
$40-44$ ans & $\mathbf{3 , 3}$ & 5,2 \\
$45-49$ ans & $\mathbf{4 , 1}$ & 5,7 \\
$50-54$ ans & $\mathbf{5 , 4}$ & 6,0 \\
$55-59$ ans & $\mathbf{8 , 6}$ & 5,6 \\
+60 ans & 2,0 & $-0,3$ \\
\hline
\end{tabular}

Source : calculé à partir de Rodosho éd. (Ministère du Travail) Rodohakusho 1988 nenpan (livre blanc sur le travail, 1988), tableau $1-5-3$, p. 187 annexe statistique.

Ces diverses catégories de salariés constituent ce que l'on peut appeler, en se référant aux théories de la segmentation du marché du travail, une main-d'oeuvre secondaire, c'est-à-dire ne bénéficiant pas des garanties du système d'emploi dominant $^{11}$ : l'emploi à vie.

A ceci s'ajoute un niveau moyen d'étude inférieur dans les PMI bien que celles-ci tentent de plus en plus de recruter quelques diplômés d'université. Le problème du niveau de formation initiale des salariés de PMI est directement connecté à l'importance des embauches de travailleurs âgés. Si peu de jeunes se présentent aujourd'hui sur le marché du travail avec 
le niveau lycée 1er cycle, bon nombre de ces travailleurs âgés en cours de carrière n'ont pas dépassé ce stade. Rien de surprenant alors que la part des salariés dont le niveau scolaire est le lycée 1 er cycle soit encore relativement élevée dans les petites firmes : les entreprises de moins de 30 salariés emploient plus de $40 \%$ des diplômés de lycée 1 er cycle, celles de 30 à 99 salariés $26,1 \%$ tandis que celles de plus de 500 n'en absorbent que 11,2\%. En ce qui concerne le niveau lycée 2ème cycle, c'est-à-dire le diplôme de fin d'études secondaire, la proportion est inverse, les moins de 30 en emploient $13,8 \%$, les 30 à 99 salariés $19,8 \%$ tandis que les plus de 500 en absorbent $35,7 \%$.

Face au changement technique, qu'il se situe au niveau des équipements introduits ou plus encore au niveau des nouvelles contraintes de gestion et d'organisation, notamment en liaison avec l'informatisation, les PMI ressentent de façon accrue le besoin de nouvelles compétences que la composition de leur main-d'oeuvre ne leur permet pas de trouver en elles-mêmes.

\subsection{Le besoin de nouvelles compétences exprimé par les PMI}

Entre 1976 et 1985, la structure de l'emploi par professionnalité a évolué de façon différenciée entre les grandes et les petites entreprises (1.023.000 personnes en moins sur la période), il augmentait fortement dans les PMI (+ 3.551.000 personnes). Dans les deux catégories d'entreprises, un effort a été fait sur les personnels spécialisés (gestionnaires, managers) et les techniciens/ingénieurs, catégories correspondant à des diplômés universitaires de cycle court ou long. Le nombre des personnels spécialisés dans le commercial s'est également accru, certes dans une moindre proportion, quelle que soit la taille des firmes. Par contre, largement différenciée, et quelque peu paradoxale est l'évolution des ouvriers qualifiés et non qualifiés. Alors que dans les petites comme dans les grandes, le nombre des ouvriers qualifiés a baissé, celui des ouvriers non qualifiés s'est largement accru 
dans les PMI (plus 979.000 contre moins 1.298.000 dans les GE). A un moment où l'élévation du niveau technique de la maind'oeuvre devient un enjeu important pour la survie des PMI, il est surprenant de constater que les ouvriers non qualifiés aient pu être privilégiés par rapport aux ouvriers qualifiés.

Il est à supposer que cette situation reflète tout d'abord le manque général de main-d'oeuvre dont souffraient les PMI dans les périodes récentes; $56,4 \%$ des PMI estimaient avoir besoin d'ouvriers qualifiés et $45,8 \%$ d'ouvriers non qualifiés. Elle reflète également la difficulté qu'ont les PMI à s'assurer la main-d'oeuvre qui leur est nécessaire, d'où le manque accumulé précédemment; la seule solution pour résoudre ce problème étant, semble-t-il, la substitution entre les travailleurs adéquats et ces laissés pour compte des grandes entreprises, qui, bien que non qualifiés, ont cependant le mérite d'avoir acquis une certaine expérience dans une grande entreprise (tableau 4). Ce manque qu'elles ont enfin pu combler en recrutant les travailleurs dont les grandes entreprises cherchaient autant que faire se peut à se décharger paraît aujourd'hui moins important. En effet, la proportion des PMI qui se plaignent encore d'un manque d'ouvriers non qualifiés est aujourd'hui minime (tableau 5). 
Tableau 4 : Evolution du nombre de salariés par profession, industrie manufacturière, 1976-1985 (unité millier de personnes)

\begin{tabular}{lrr}
\hline & PME & GE \\
\hline & 3.551 & -1.023 \\
Total & 1.852 & 1.222 \\
gestionnaires, managers & & \\
techniciens/ingénieurs & 250 & -325 \\
employés de bureau & 482 & 256 \\
personnel commercial & 18 & -372 \\
personnel de transport et & & -881 \\
communication & -117 & -1.298 \\
ouvriers qualifiés & 979 & 577 \\
ouvriers non qualifiés & 32 & -203 \\
employés de service & 55 & \\
autres & & \\
\hline
\end{tabular}

Source : livre blanc sur les PME, 1987, p. 102. 
Tableau 5 : Surplus ou manque de main-d'oeuvre par professions. unité : pourcentage d'entreprise ayant répondu "manque" moins pourcentage d'entreprises ayant répondu "surplus"

\begin{tabular}{lrr}
\hline & PME & GE \\
\hline & & \\
toutes professions confondues & 19,4 & $-21,1$ \\
techniciens/ingénieurs de production & 44,9 & 45,1 \\
personnel de recherche et développement & 64,1 & 73,3 \\
ouvriers qualifiés & 34,2 & 3,1 \\
ouvriers non qualifiés & 1,3 & $-31,3$ \\
personnel commercial & 37,8 & 29,9 \\
personnel de planification & 43,8 & 36,3 \\
personnel financier, comptabilité & 16,0 & 11,8 \\
personnel de gestion & 9,4 & $-14,9$ \\
\hline
\end{tabular}

Source : livre blanc PME 1987, p. 103.

Quant aux prévisions sur les besoins de demain, si un pourcentage encore relativement important de PMI considèrent que les travailleurs de production, qualifiés et non qualifiés, seront encore d'une grande nécessité, une fraction encore plus grande d'entre elles considère que leurs besoins se tourneront principalement vers les techniciens et ingénieurs de production, les chercheurs et personnels capables de mener à bien des projets de recherche et développement, ainsi que, bien qu'à un degré moindre, du personnel commercial et des hommes compétents pour la planification et la mise en oeuvre d'opérations d'informatisation (tableau 6). En ce qui concerne les chercheurs et techniciens/ingénieurs de production, les besoins prévus par les grandes entreprises sont encore plus conséquents, il est donc fort probable que les PMI auront des difficultés à recruter ce type de main-d'oeuvre. 
Tableau 6 : Evolution des besoins de main-d'oeuvre des entreprises de l'industrie manufacturière

unité : pourcentage d'entreprises

\begin{tabular}{lrrrrr} 
& \multicolumn{2}{l}{ jusqu'à présent } & \multicolumn{2}{c}{ années à venir } \\
\cline { 2 - 6 } & PME & GE & PME & GE \\
\cline { 2 - 5 } & & & & $\mathbf{3 6 , 3}$ & $\mathbf{1 6 , 2}$ \\
ouvriers qualifiés & 56,4 & $\mathbf{4 1 , 4}$ & $\mathbf{2 2 , 0}$ & $\mathbf{8 , 1}$ \\
ouvriers non qualifiés & 45,8 & 32,3 & $\mathbf{4 2 , 8}$ & $\mathbf{6 8 , 7}$ \\
tech./ingénieurs production & 31,8 & 69,7 & $\mathbf{2 8 , 5}$ & $\mathbf{2 5 , 3}$ \\
commercial & $\mathbf{2 7 , 1}$ & $\mathbf{2 3 , 2}$ & $\mathbf{4 2 , 1}$ & $\mathbf{7 7 , 8}$ \\
recherche développement & 24,1 & 64,6 & $\mathbf{2 2 , 5}$ & $\mathbf{3 4 , 3}$ \\
planif.,informatisation & 9,4 & 16,2 & $\mathbf{1 2 , 6}$ & $\mathbf{9 , 1}$ \\
finance comptabilité & 9,1 & 7,1 & $\mathbf{2 , 0}$ & $\mathbf{3 , 0}$ \\
autres & 0,8 & 1,0 & $\mathbf{0 , 0}$ & $\mathbf{0 , 0}$ \\
pas de besoins particuliers & 9,5 & 5,1 & &
\end{tabular}

Source : livre blanc sur les PME, 1987 p. 203.

1.3. La recherche des nouvelles compétences à l'extérieur

Compte tenu des besoins exprimés, et du type de maind'oeuvre disponible dans les PMI (femmes, travailleurs d'âge moyen ou élevé, laissés pour compte des GE), quels sont les moyens à la disposition des PMI pour trouver, ou développer en interne, les compétences requises?

Les stratégies en matière de recrutement

Face au constat des besoins qu'elles se doivent de satisfaire dans les années à venir, un nombre d'entreprises, d'autant plus élevé que leur taille est grande, ont élaboré une politique d'embauche sur plusieurs années. Ainsi, plus de $90 \%$ des firmes de plus de 1.000 salariés ont planifié avec précision leurs besoins 
de recrutement contre $74 \%$ de celles de 100 à 299 et $52 \%$ de celles de 30 à 99 salariés $^{12}$. La lecture de ces plans d'embauche donne une assez bonne illustration des problèmes qui se posent et des stratégies qui vont être déployées en matière d'emploi. Exprimés en pourcentage d'entreprises qui comptent mettre en oeuvre ou accroître telle ou telle action, ils ne permettent pas de saisir l'importance en terme de nombre de salariés concernés; ainsi, lorsqu'une PMI affiche la volonté d'augmenter ses diplômés d'université, il peut s'agir du recrutement d'une ou deux personnes alors que dans le cas d'une grande entreprise, le nombre serait multiplié par 10 . Néanmoins, et même si l'existence d'un plan d'embauche ne conditionne pas la réussite des actions entreprises, il y a là un certain nombre d'informations intéressantes.

Parmi les principales directions définies dans la politique d'embauche des entreprises concernées, toutes ne visent pas à résoudre des problèmes du même type. Ainsi, un pourcentage important de firmes, grandes mais aussi petites, entendent recruter quand même un minimum de jeunes à la sortie de l'école, afin de ne pas accentuer le déséquilibre de leur pyramide des âges ${ }^{13}$ :

$\begin{array}{ll}+5.000 \text { sal. } & 51,5 \\ 1.000 \text { d } 4.999 & 53,4 \\ 300 \text { \ } 999 & 52,7 \\ 100 \text { a } 299 & 45,6 \\ 30 \text { a } 99 & 36,8\end{array}$

Par contre, un pourcentage nettement inférieur et qui ne semble pas être directement lié à la taille des entreprises, compte profiter, pour limiter les embauches de salariés réguliers, des possibilités d'emploi à temps partiel (en moyenne toutes tailles confondues : $19 \%$ ), ou du prolongement de l'âge de la retraite $(6,8 \%)$ ou encore du prêt de main-d'oeuvre par le 
donneur d'ordres pour les petites ou par les sous-traitants pour les plus grandes $(10,1 \%)$.

Les autres éléments mis en avant dans les plans d'embauche concernent davantage les évolutions qualitatives actuelles. En premier vient l'embauche de techniciens et d'ingénieurs pour l'introduction de nouvelles technologies et l'amélioration des capacités de recherche et développement. La proportion d'entreprises qui comptent oeuvrer dans ce sens diminue en fonction de la taille mais reste cependant élevée pour les PMI :

$\begin{array}{ll}+5.000 \text { sal. } & 94,9 \\ 1.000 \text { a } 4.999 & 87,1 \\ 300 \text { व } 999 & 68,7 \\ 100 \text { a } 299 & 54,2 \\ 30 \text { a } 99 & 37,5\end{array}$

Plus largement prise en compte par les grandes entreprises, l'embauche de spécialistes pour la pénétration de nouveaux domaines n'est pas une préoccupation complètement absente des PMI :

$\begin{array}{lr}+5.000 \text { sal. } & 65,4 \\ 1.000 \text { d } 4.999 & 50,4 \\ 300 \text { à } 999 & 36,7 \\ 100 \text { d } 299 & 23,2 \\ 30 \text { à } 99 & 19,1\end{array}$

Par contre, l'embauche de travailleurs d'un niveau de fin de secondaire pour renforcer l'atelier est davantage un problème de PMI (6,6\% des entreprises de plus de 5.000 sal. contre $23,3 \%$ de celles de 100 à 299) et va tout à fait dans le sens de ce qui a déjà été mentionné.

De même, mais en sens inverse, l'élargissement des domaines d'activité des femmes est davantage prôné par les grande sentreprises $(44,9 \%$ des plus de 5.000 contre $18,7 \%$ des 
100 ) 299), confirmant également les tendances d'avenir décrites précèdemment.

Pour répondre à ces orientations, vers quelles catégories de main-d'oeuvre les entreprises vont-elles se tourner ? L'enquête à laquelle il est fait référence ici distingue les emplois de bureau et de gestion, les emplois techniques et les emplois de production $^{14}$.

En ce qui concerne les emplois de bureau et de gestion, les grandes entreprises se proposent d'embaucher principalement des diplômés d'université, hommes ou femmes, cycle long de préférence mais aussi cycle court pour les tâches "nobles" et des salariés à temps partiel pour le reste. Les moyennes entreprises pensent se tourner davantage vers des hommes, diplômés d'université, cycle long puis court, et également vers le temps partiel, tandis que les petites penchent pour des femmes ayant le niveau d'études secondaires, des hommes diplômés d'université de cycle long, et un recours nettement inférieur au temps partiel. Il faut rappeler que cette catégorie de firmes absorbe déjà une part conséquente de cette forme d'emploi.

Pour ce qui est des emplois techniques, ce sont les diplômés d'université qui sont largement privilégiés par toutes les catégories d'entreprises, mais alors que la plupart des PMI pensent recruter des hommes, le pourcentage de grandes entreprises qui envisagent de se tourner vers des femmes est significatif d'une tendance nouvelle.

Au niveau des emplois de production, la situation est beaucoup moins tranchée, les entreprises se répartissant entre la volonté d'embauche de diplômés du lycée deuxième cycle et de diplômés d'université cycles court ou long, hommes ou femmes. Il apparaît toutefois une décroissance des entreprises favorisant l'université au fur et à mesure que l'on descend dans 
la taille, baisse d'autant plus marquée qu'il s'agit de cycle court et surtout des femmes. Les emplois pour lesquels les PMI veulent recruter des diplômés d'université sont des postes de responsabiltié (ce qui n'est pas forcément le cas des GE) qu'elles ne peuvent, la pression sociale aidant, confier qu'à des hommes.

Le pourcentage d'entreprises, y compris de petite taille, qui ont mis au point une politique d'embauche ne doit pas faire oublier que l'adaptation de la main-d'oeuvre aux nouvelles contraintes ne peut se faire exclusivement par cette voie, notamment dans un système qui privilégie l'attachement d'un salarié à une seule entreprise. Certes, ce système tend à être, petit à petit, modifié aux frontières, mais on est en droit de se demander quelle est la marge de manoeuvre des PMI pour mener à bien la politique présentée dans leurs plans d'embauche.

Les autres possibilités d'apport de main-d'oeuvre extérieure

Le recrutement direct de jeunes sortant du système éducatif, notamment au niveau de l'université est, comme cela a déjà été mentionné, difficile pour les PMI qui d'une part, entrent alors en concurrence directe avec les GE et d'autre part, ne peuvent soutenir les coûts de recrutement que cela implique. Il est donc probable que, pour s'assurer la main-d'oeuvre décrite ci-dessus, de plus en plus de PMI devront se tourner vers des recrutements en dehors des campagnes de printemps ${ }^{15}$. Mais même si les opportunités de recrutements en cours de carrière augmentent du fait d'un certain effritement du système d'emploi dominant, il semble difficile qu'elles puissent ainsi combler l'ensemble de leurs besoins de nouvelles compétences. D'autres procédures d'apport extérieur de main-d'oeuvre existent, qui pourraient être des solutions temporaires, mais paradoxalement les PMI en font peu usage. 
Lié à la volonté d'externaliser certaines fonctions comme par exemple l'entretien des bâtiments, ou pour faire face à un problème de manque d'effectifs ou de compétences particulières, notamment en informatique, le recours à une main-d'oeuvre détachée d'une autre entreprise (haken rodosha) s'est répandu largement, surtout dans les grandes entreprises; $79,4 \%$ des plus de 5.000 salariés, ou encore $50 \%$ des 300 à 999 salariés contre seulement $14,8 \%$ des 30 à 99 salariés (tableau 7).

Tableau 7: Pourcentage d'entreprises utilisant de la main-d'oeuvre détachée d'une autre entreprise

moyenne informatique gestion bâtiment sans recours

\begin{tabular}{lrrrrr}
+5.000 sal. & 79,4 & 56,0 & 43,6 & 52,8 & 20,6 \\
$1.000-4.999$ & 71,1 & 44,6 & 30,4 & 45,6 & 28,9 \\
$300-999$ & 50,0 & 24,5 & 20,3 & 32,5 & 50,0 \\
$100-299$ & 27,3 & 9,6 & 10,7 & 15,5 & 72,7 \\
$30-99$ & 14,8 & 3,0 & 6,7 & 8,0 & 85,2 \\
moyenne enquête & 20,6 & 6,5 & $\mathbf{8 , 8}$ & 11,8 & $\mathbf{7 9 , 4}$ \\
\hline
\end{tabular}

Source : Rodo daijin kanbo seisaku chosabu éd., 1986, p. 68.

Il reste une certaine ambiguité en ce qui concerne le statut de ces personnels "détachés" qui semble regrouper le travail en régie, les prestataires de service... Une analyse juridique serait à mener, parallèlement à l'étude du travail intérimaire (haken gyo) permis tout récemment ${ }^{16}$.

Autorisé depuis relativement peu de temps pour certaines tâches (loi de 1986), l'intérim est présentée comme une solution offerte pour tenter d'adapter savoir-faire interne aux compétences requises. Le recours à ce type de service est encore, comparativement aux grandes entreprises $(25,7 \%)$, limité dans 
les PMI $(2,9 \%)$, mais il semble qu'il soit appelé à se développer dans les années à venir, puisque $17,1 \%$ de GE et $13,3 \%$ de PMI qui ne s'en servaient pas, comptent dorénavant le faire. Les PMI qui l'utilisent le font principalement pour répondre à un manque de personnel $(47 \%)$ ou parce que la formation du personnel prend du temps alors qu'il y a urgence (44\%). Les PMI qui ne s'adressent pas aux agences d'intérim disent préférer former leurs salariés pour accroître l'accumulation de savoir-faire interne $(40 \%)$ ou que la formation en interne de leurs salariés est moins coûteuse $(28 \%)$. Dans les raisons invoquées, apparaît clairement l'attachement des entreprises japonaises à la formation interne, considérée comme un investissement humain. Apparaît également le problème du coût de l'intérim.

Quelles que soient les stratégies de recrutement des PMI, et même si leur degré de réussite dans cette voie est optimal, il est évident que l'acquisition des nouvelles compétenes qui leur sont indispensables pour survivre dans les années 90 ne pourra se limiter au recours à une main-d'oeuvre extérieure. Les capacités d'embauches directes ne peuvent pas suffire à combler le déficit dû au handicap de départ et à leur position défavorable dans la concurrence avec les GE. Les modalités plus récentes, telles que le travail intérimaire, ne sont pas encore vraiment entrées dans les moeurs des PMI qui semblent rebutées par le coût que cela implique. Même si ces formes d'emploi venaient à se répandre dans les années à venir, elles ne pourraient se substituer durablement et totalement à la nécessité d'accroître le niveau de compétence interne par la formation du plus grand nombre. Les PMI en sont également conscientes, et le fait qu'elles aient élaboré des plans d'embauche ne signifie pas qu'elles renoncent à la volonté de former leurs salariés. Bien au contraire, le recrutement d'un diplômé d'université est souvent vu comme un moyen d'internaliser les connaissances nécessaires à une diffusion en 
cascade par les mécanismes habituels de formation interne.

2. L'acquisition des nouvelles compétences et les procédures de formation

La formation professionnelle tient une place importante dans les entreprises japonaises, y compris de petite taille. Certes, les PMI privilégient largement la formation sur le tas pour le plus grand nombre de leurs salariés, mais elles disposent également de diverses procédures qui permettent un réel transfert de compétences émanant de leurs donneurs d'ordres. Le changement technique n'a pas affecté le fonctionement de ces mécanismes de formation qui ont au contraire tendance à se renforcer, mais l'évolution du contenu du travail vers une plus grande complexité et l'apparition de nouvelles fonctions entrainent un besoin accru de formations pour lesquelles les procédures habituelles, bien qu'elles ne soient pas rendues caduques, s'avèrent insuffisantes. Ainsi apparaissent, aux côtés des modalités "anciennes" de formation des salariés, des formes "nouvelles" d'acquisition des compétences.

Or, si la quasi totalité des entreprises offrent à leurs salariés une formation, non seulement à l'entrée, mais aussi tout au long de leur carrière (tableau 8), le pourcentage de la masse salariale qui lui est consacrée dans les statistiques officielles, tout comme les chiffres annoncés par les entreprises, sont extrêmement faibles (tableau 9). Il y a là un paradoxe qu'il convient d'expliquer. 
Tableau 8 : Pourcentage d'entreprises offrant une formation à leurs salariés

\begin{tabular}{lcc}
\hline taille entreprise & offrant formation & n'offrant pas formation \\
\hline 1.000 sal. et + & 99,0 & 1,0 \\
500 a 999 sal. & 96,2 & 3,8 \\
300 a 499 sal. & 95,9 & 4,1 \\
100 a 299 sal. & 89,6 & 10,4 \\
30 a 99 sal. & 77,6 & $\mathbf{2 2 , 4}$ \\
\hline
\end{tabular}

Source : Japanese working life profile : statistical aspects, the Japan Institute of Labour, 1987, p. 66.

Tableau 9 : Part des frais de formation dans la masse salariale

\begin{tabular}{lc}
\hline Entreprises & Industrie manufacturière \\
\hline moyenne & 0,4 \\
& \\
+5.00 sal. & 0,7 \\
1.000 a 4.999 sal. & 0,4 \\
300 a 999 sal. & 0,3 \\
100 d 299 sal. & 0,2 \\
30 a 99 sal. & 0,2 \\
\hline
\end{tabular}

Source : Rodosho Seisakuchosabu éd., 1987, p. 128-133.

La formation est d'abord une affaire interne qui irrigue en permanence la vie de l'entreprise. Son coût est souvent comptabilisé en frais généraux car elle ne constitue qu'un élément parmi d'autres dans l'objectif poursuivi (équipes de 
projet...), ou passe par des mécanismes institutionnalisés (rotations...) qui en dépassent largement le cadre.

Cela n'exclut pas le recours ponctuel à des formations extérieures dont le rôle est loin d'être négligeable. La distinction entre interne et externe est d'ailleurs souvent floue. Il existe diverses procédures externes à l'entreprise mais internes au système productif qui, par le biais de l'information et de la circulation des hommes, contribuent incontestablement à leur formation. C'est le cas, par exemple, des relations entre donneurs d'ordres et fournisseurs/sous-traitants qui constituent un vecteur important de formation en cascade. Toute cette dynamique n'apparaît pas dans les coûts de formation officiels. Au cours d'entretiens menés en entreprises, un certain nombre d'estimations ont pu être obtenues. Toutes sont cependant très inférieures à la réalité. En effet, lorsque poursuivant la discussion, il était question des nombreuses allées et venues de personnels entre le donneur d'ordres et les fournisseurs/sous-traitants, il est apparu clairement que le coût de ces mouvements n'entrait jamais dans les estimations faites. C'est donc une masse d'heures de formation qui échappent ainsi à tout comptabilisation. Ainsi, des opérations dont la finalité première n'est pas la formation mais qui y contribuent grandement ne sont pas prises en compte dans les budgets spécifiques.

Qu'elles aient ou non un lien avec l'introduction de la micro-électronique, qu'elles touchent tous les salariés (formation à l'entrée) ou seulement une partie d'entre eux (formation continue), la prépondérance de l'interne (entendu au sens de système productif) sur l'externe expliquerait donc la faiblesse des coûts de formation affichés par les entreprises japonaises. Cette situation pourrait être amenée à changer si des formules nouvelles de type plus occidental, comme le recours à des consultants spécialisés, venaient à se répandre. 
C'est ce qui semble timidement se mettre en place à travers l'exemple du plan Icho dont il sera question ultérieurement. Il est toutefois encore trop tôt pour se prononcer à ce sujet même si un ferment semble être présent.

3.1. Face au changement technique, quelle formation et pour qui ?

Traditionnellement, plus de la moitié des PMI de l'industrie manufacturière se contentent d'une formation sur le tas $(57,2 \%$ des PMI) alors que les grandes entreprises, tout en donnant une place centrale à la formation sur le tas "on the job training" (OJT), ont majoritairement tendance à lui associer une formation hors travail ou "off the job training" (Off JT) $(78,1 \%$ des GE).

En liaison avec le changement technique, un pourcentage important de PMI constatent cependant que la formation sur le tas n'est plus suffisante mais qu'elles n'ont pas les moyens de faire de la formation hors travail : 
Tableau 10 : Formation sur le tas, formation hors travail : la proposition des PMI de l'industrie manufacturière pour les années a venir (\% d'entreprises)

\begin{tabular}{lcc}
\hline & PME & GE \\
\hline $\begin{array}{l}\text { OJT n'est pas suffisante mais pas les } \\
\text { moyens de Off JT }\end{array}$ & 45,5 & 27,8 \\
$\begin{array}{l}\text { OJT suffit, OFF JT inutile } \\
\begin{array}{l}\text { OJT n'est pas suffisante } \\
\text { Off JT envisagée }\end{array}\end{array}$ & 23,3 & 0,0 \\
$\begin{array}{l}\text { OJT suffit mais Off Jt envisagée } \\
\text { pour accroître efficacité de la } \\
\text { formation }\end{array}$ & 7,4 & 27,8 \\
\hline
\end{tabular}

Source : Livre blanc sur les PMI, 1987, p. 206.

Une enquête du National Institute of Employement and Vocational Training (NIEVR) ${ }^{17}$ confirme qu'en ce qui concerne l'introduction d'équipements de micro-électronique notamment, les PMI n'ont pas le potentiel interne pour se limiter à de l'OJT. La formation sur le tas sous-entend, en effet, qu'il y ait dans l'entreprise un minimum de compétences à transmettre. Elles doivent donc impérativement s'adresser à l'extérieur; principalement représenté en matière de formation/adaptation aux équipements nouveaux par le fabricant de ces équipements (tableau 11). 
Tableau 11 : Types de formation développés lors de l'introduction d'équipements

\begin{tabular}{|c|c|c|c|c|c|c|c|c|c|c|c|c|c|c|c|}
\hline $\begin{array}{c}\text { Taille } \\
\text { d'entreprise }\end{array}$ & $\begin{array}{l}\text { echan-l } \\
\text { tilianl }\end{array}$ & 1 & 2 & 3 & 4 & 5 & 6 & $?$ & 8 & 9 & 10 & 11 & 12 & 13 & 14 \\
\hline Total & 1290 & 981 & 880 & 102 & 100 & 285 & 502 & 176 & 154 & 521 & 190 & $i 3$ & 409 & 10 & 12 \\
\hline (en $x$ & 100.0 & 76.0 & 68.2 & 7.9 & 7.8 & 22.1 & 38.9 & 13.6 & 11.9 & $4 \dot{v}, 4$ & 14.7 & $5 . i$ & 31.7 & 0.8 & 0.9 \\
\hline < 99 sal. & 181 & 76.2 & 68.0 & 7.2 & 8.8 & 14.4 & 23,2 & 6.6 & 3.3 & 24.9 & 12.2 & 1.7 & 35.4 & 1.1 & 2.8 \\
\hline $100+199$ & 378 & 76.2 & 65.9 & 9,0 & 9.3 & 20.6 & 29.9 & 11.9 & 8.2 & 36.5 & 10.3 & 4.8 & 31.2 & 0.8 & 0.5 \\
\hline $200 \propto 299$ & 174 & 78.7 & 74.7 & 5.2 & 7.5 & 14.9 & 39.1 & 8.6 & 10.9 & 35,6 & 12.1 & 4.6 & 25.9 & 0.6 & 1.1 \\
\hline $300+499$ & 196 & 71,9 & 68.4 & 7,7 & 6.1 & 25.5 & 46,4 & 14.8 & 16.8 & 39.3 & 15.6 & 5,1 & 34.7 & 1,0 & 0.5 \\
\hline $500+999$ & 188 & 76.6 & 67,6 & 10.6 & 8,5 & 26.1 & 48,9 & 17.0 & 16 & 48.4 & 19.1 & 8.0 & 34.0 & 0.5 & 0.5 \\
\hline 1000 & 173 & 76.9 & 67,6 & 6. & 4.6 & 32.4 & 55.5 & 24.9 & 20.2 & 62,4 & 23.7 & 11.0 & 28.9 & 0.6 & 0.6 \\
\hline
\end{tabular}

Source : NIEVR, 1985, p. 147.

1 = formation chez le fabricant

2 = formation dans l'entreprise par du personnel du fabricant

3 = formation auprès d'organismes privés extérieurs

4 = formation auprès d'organismes publics extérieurs

$\mathbf{5}$ = formation dans le centre de formation propre de l'entreprise

$\mathbf{6}=$ formation par participation aux tests de lancement

7 = formation par participation à l'équipe de projet

$8=$ OJT dans un autre atelier plus avancé

9 = OJT par des techniciens ou des personnels expérimentés de l'entreprise

$\mathbf{1 0}$ = formation par apprentissage de la maîtrise de plusieurs machines

11 = formation par correspondance

12 = formation individuelle par soi-meême (audomicile par ex.)

13 = pas de formation particulière

14 = sans réponse 
Pour chaque machine achetée, un stage gratuit, de deux jours à une semaine, est proposé par tous les fabricants pour deux ouvriers. Il est rare qu'une entreprise n'achète qu'une seule machine. Il y a donc un effet multiplicateur du nombre de personnes ainsi formées. Celles-ci apprennent ensuite à d'autres opérateurs à utiliser les machines par le principe de la doublure ce qui constitue un retour à l'OJT mais dans un deuxième temps. Dans certains cas, les opérateurs sélectionnés vont chez le fabricant avant que la machine ne soit livrée et participent ainsi plus efficacement à l'installation sur leur lieu de travail. Dans de nombreux cas, le stage est d'ailleurs assuré par les ingénieurs du fabricant dans les ateliers même de l'entreprise utilisatrice. Le recours au stage utilisateur des fabricants d'équipements n'est certes pas limité aux PMI mais, alors que les grandes entreprises ont la capacité interne à lui associer un complément de formation, les PMI n'ont parfois que cette possibilité pour aborder l'automatisation. Ce qui vient d'être dit à propos des équipements de micro-électronique se reproduit à peu près à l'identique pour l'informatisation, le concepteur du logiciel jouant le même rôle que le fabricant des machines.

Qu'il s'agisse de l'introduction de nouvelles machines, ou de la mise en oeuvre de changements organisationnels, tous les salariés ne sont pas concernés de la même façon. Toujours à travers le cas de la micro-électronique, il est possible de cerner les critères de sélection mis en avant par les entreprises.

Profil des salariés chargés des équipements de microélectronique

La sélection commence au stade de la formation qui est nécessaire pour assumer les nouvelles fonctions. La prise en charge des équipements de micro-électronique n'est effectivement pas ouverte à tous de façon identique. Le 
problème spécifique des travailleurs âgés est d'emblée posé. Un processus d'exclusion par l'âge joue en effet dans la grande majorité des entreprises, y compris de petite taille.

Tableau 12 : Age des salariés sélectionnés pour formation

\begin{tabular}{lrrrrrr}
\hline & -24 ans & $25-29$ & $30-34$ & $35-39$ & $40-44$ & +45 \\
\hline$<99$ & 20,1 & 30,2 & 25,2 & 17,6 & 3,8 & 1,9 \\
$200-999$ & 18,0 & 30,2 & 25,2 & 17,6 & 3,8 & 1,9 \\
$>1000$ & 8,1 & 40,9 & 36,2 & 13,4 & 0,7 & 0,0 \\
\hline
\end{tabular}

Source : NIEVR, 1985, p. 154.

Les travailleurs concernés par la micro-électronique sont donc plutôt jeunes, ils ont le niveau baccalauréat et sont principalement ouvriers de production. Ils ont généralement une solide expérience professionnelle en manuel.

Tableau 13: Formation initiale des ouvriers de production chargés des équipements ME (\% d'entreprises)

\begin{tabular}{lcccc}
\hline Lycée 1 & Lycée 2 & $\begin{array}{c}\text { Université } \\
\text { cycle court }\end{array}$ & $\begin{array}{c}\text { Université } \\
\text { cycle long }\end{array}$ \\
$<99$ & 23,2 & 75,1 & 6,1 & 8,8 \\
$200-299$ & 13,2 & 85,6 & 5,2 & 0,6 \\
$>1000$ & 14,5 & 90,8 & 3,5 & 2,3 \\
\hline
\end{tabular}

Source : NIEVR, 1985, p. 111. 
Tableau 14 : Expérience en manuel des ouvriers de production chargés des équipements ME (\% d'entreprises)

\begin{tabular}{lrcc}
\hline & & & \\
& aucune & $5-9$ ans & $10-19$ ans \\
\hline 39 & 12,2 & 32,0 & 38,0 \\
$200-299$ & 13,2 & 44,8 & 33,3 \\
$>1000$ & 8,1 & 48,0 & 45,1 \\
\hline
\end{tabular}

Source : NIEVR, 1985, p. 115.

Comme le fait justement remarquer C. Mercier, ces caractéristiques appellent les interprétations suivantes :

- La formation s'appuie sur l'expérience professionnelle traditionnelle, elle ne la remplace pas. Les entreprises japonaises ont compris que les savoir-faire nouveaux (avec équipements micro-électroniques) ne peuvent s'acquérir que sur la base et par incorporation des savoir-faire anciens. Une pratique professionnelle préalable est indispensable pour accéder à la maîtrise de technologies supérieures.

- La formation n'exclut pas les ouvriers de production, elle s'adresse au contraire principalement à eux, compte tenu des interventions qui leur sont demandées ${ }^{18}$.

Cette formation peut se limiter à un simple apprentissage en doublure sur le poste, ou à une formation sur le tas dispensée par le chef d'équipe ou un technicien, ou encore par le fils du PDG dans de petites entreprises. Il est en effet fréquent dans cette catégorie de formes que le fils du PDG ait étudié à l'université, voire parfois été employé dans une GE quelques 
années, avant d'apporter à l'entreprise familiale le savoir- faire ainsi accumulé. Il joue lors, entre autre, le rôle de formateur sur le tas, intervenant partout où cela est nécessaire jusqu'à ce que d'autres puissent prendre le relais.

La formation aux nouvelles technologies ne se limite pas à l'apprentissage du fonctionnement d'une nouvelle machine, d'autant plus que l'intégration des équipements est croissante et que les changements organisationnels sont de plus en plus importants. Dans ces différents cas, lorsque la formation associe OJT à Off JT, elle peut rester totalement interne et passer par les mécanismes habituels de formation. Il peut s'agir, par exemple, de la participation aux cours pour nouveaux embauchés dans la partie liée à l'automatisation ou à la gestion de la production. Des exercices pratiques sur le tas ont toujours lieu simultanément. Des stages spécifiques sont parfois élaborés dans l'entreprise dans la phase de préparation d'un investissement. Dans ce dernier cas dont l'équipe de projet est le meilleur exemple, un recours à l'extérieur peut éventuellement s'avérer nécessaire.

\subsection{La formation par participation à la conception/réalisation de l'investissement}

C'est de loin la forme la plus élaborée des stratégies indirectes de formation mises en oeuvre dans les entreprises. Elle suppose généralement l'existence d'une structure de fabrication ou au moins d'adaptation en interne de machines, même si la plupart des éléments sont achetés à l'extérieur. Elle passe en principe par la constitution d'une équipe de projet.

Qu'il s'agisse d'un projet d'investissement de substitution ou de la préparation d'une nouvelle gamme de produits, certaines entreprises japonaises constituent dès le point de départ une équipe de projet chargée d'instruire le dossier. Cette équipe de projet n'implique pas les mêmes personnes tout au long de son fonctionnement qui peut durer plusieurs années 
pour les investissements lourds. Ainsi, une entreprise de 385 salariés avait déjà fin 1983 constitué une équipe de projet pour réfléchir à l'automatisation complète de son atelier de fonderie prévue pour $1986^{19}$.

\section{Le modèle "quasi-parfait" de l'équipe de projet}

La démarche part généralement des services fonctionnels hors de la production. Le service de planification des prix de revient (ou son homologue) a pour fonction de se tenir au courant des évolutions en matière d'équipements. C'est donc lui qui en fonction des possibilités techniques existantes fait le premier diagnostic en terme de coût/rentabilité des choix d'investissement qui sont ouverts. C'est sur ces bases que va travailler l'équipe de projet. Dès cette première phase sont associés au projet les techniciens de la division ou du service technique qui ont en charge la conception des machines fabriquées en interne. S'il n'est pas exclu que les équipements soient finalement achetés à l'extérieur, une analyse est faite, option par option entre ce qu'elle implique d'achats et ce qu'elle permet de réalisations internes. Certaines entreprises ont pour principe d'acheter les équipements lorsque la réalisation maison s'avère trop coûteuse, trop complexe ou trop longue. D'autres préfèrent "fabriquer maison" tout ce qui est techniquement possible même si le délai d'exécution est plus long, à condition qu'il y ait un avantage de coût.

Est également associé dès ce stade le chef d'usine lorsqu'il s'agit d'un groupe, et le chef d'atelier qui aura à superviser l'installation, qu'il s'agisse d'une simple ligne ou de tout un atelier. Participent aussi les personnels commerciaux chargés de la prospection des fabricants possibles pour la part d'équipements clef en main. L'équipe de projet ainsi composée fait les études techniques incluant la fiabilité, l'entretien etc... et les études chiffrées de coût d'investissement et de coût 
d'exploitation. Elle visite les fabricants de $\mathrm{MO}$, des foires et surtout lorsqu'un équipement du même type existe quelque part, elle fait des visites d'utilisateurs.

A partir du moment où une solution est retenue, la démarche va inclure de plus en plus de personnel de la production proprement dite dans l'équipe de projet. La division ou le service technique va prendre le relais du service de planification des prix de revient pour la maîtrise d'oeuvre du projet. Les concepteurs de machines en interne se mettent à l'oeuvre, associant les réalisateurs de ces équipements (service ingénierie/outillage) et les personnes chargées du suivi de la fabrication à l'extérieur (lorsqu'il ne s'agit pas d'un achat sur catalogue). Dans cette même phase, l'équipe de projet s'adjoint le service du personnel afin de prévoir les modifications à apporter en matière d'organisation du travail. Des transferts sont envisagés soit dans l'atelier, soit sur plusieurs ateliers. Dans ce cas, les chefs d'ateliers concernés sont associés à l'équipe. A ce stade, il ne s'agit que de prévisions grossières mais elles ont pour but d'élaborer le plan de formation qu'il faut mettre en oeuvre dès le début de l'opération afin que tout soit prêt à temps. Par exemple, si l'on reprend le cas cité, la formation a porté dès 1984 sur l'apprentissage de la programmation par les opérateurs retenus pour travailler sur les nouvelles installations.

Au fur et à mesure que le projet progresse, d'autres salariés sont amenés à participer aux travaux de l'équipe de projet. Les plans terminés, la fabrication en interne des machines ou dispositifs prévus commence, et ce sont alors les chefs d'quipe, les chefs de ligne et les opérateurs pressentis pour occuper les futurs postes qui interviennent. 
L'effet formateur de l'équipe de projet

L'association des opérateurs au stade final joue un rôle important en matière de formation car s'ils ont participé au montage, ils peuvent ensuite entretenir et réparer.

Mais les aspects formateurs de l'équipe de projet ne se limitent pas à l'existence d'une structure de fabrication des équipements en interne qui peut très bien jouer le même rôle sans le support d'une telle équipe. Par le contact avec les autres membres de l'équipe, chacun apprend à parler le même langage, les opérateurs élèvent leur niveau technique et accroissent leur polyvalence. Les techniciens apprennent les petits "trucs" qui relèvent de savoir-faire empiriques que détiennent les opérateurs. Cette part de formation est totalement informelle, mais non négligeable pour autant. L'équipe de projet a par ailleurs le mérite d'associer dès le début de l'opération les responsables hiérarchiques de la production à la définition des programmes de formation qui sont nécessaires au bon fonctionnement des nouveaux équipements. Loin de se sentir dépossédés de leurs attributions, ils oeuvrent au contraire à la réussite de l'opération, en assurant souvent eux mêmes une partie de cette formation.

S'il est probable qu'une démarche aussi complète que le cas présenté n'existe que rarement dans les petites entreprises, il convient toutefois de signaler que dans un fonctionnement partiel, l'équipe de projet n'est pas l'apanage des seules grandes firmes. D'après l'enquête du NIEVR déjà citée (p. 147), le pourcentage d'entreprises de 100 à 299 salariés qui utilisent, entre autres, cette méthode est de $11,9 \%$, et celui des moins de 99 salariés de $6,6 \%$. Dans ces entreprises, l'équipe de projet peut faire intervenir l'extérieur pour lever des blocages dus au manque de compétences sur tel ou tel aspect. Ceci se fait soit par envoi de personnels dans des écoles spécialisées, soit plus 
récemment par l'assistance de "facilitateur" dans le cadre de programmes régionaux qui se mettent en place (cf. plan Icho ciaprès).

\subsection{Le recours aux organismes extérieurs ou intervenants spécialisés}

Il ne sera question que de la formation auprès d'organismes extérieurs spécialisés auxquels sont envoyés des salariés d'entreprises n'ayant pas rompu le contrat de travail avec celles-ci. L'objectif se limite ici à l'analyse des politiques d'entreprises en matière de formation et ne porte donc pas sur les politiques nationales de reconversion et d'adaptation de la population active au changement technico-organisationnel d'aujourd'hui, bien que cela occulte un certain nombre d'actions de formation qui, en définitive, bénéficient aux entreprises puisqu'elles peuvent embaucher, à l'issue de ces stages, des travailleurs mieux formés.

L'importance de la formation interne pour les entreprises japonaises a été maintes fois soulignée, au risque de laisser supposer que la formation externe n'était qu'un cas d'exception. Or comme cela a été précèdemment mentionné, les PMI ont de plus en plus conscience que la formation interne sur le tas ne suffira plus dans les années à venir. Elles sont rarement en mesure de se lancer seules dans des formations hors travail et doivent donc se tourner vers l'extérieur pour acquérir les compétences qui leur font défaut. Le recours à la formation externe n'est donc pas négligeable, tant qualitativement que quantitativement. Il est certes plus ponctuel et ne touche que quelques personnes à la fois mais il permet de lever des blocages qui ne pourraient l'être autrement et rendraient la formation interne moins performante, si ce n'est caduque. En effet, la dynamique de formation interne prend immédiatement le relais de la formation externe pour l'élargissement au sein de l'entreprise des compétences acquises par quelques uns. 
Les organismes de type chambres de commerce et d'industrie sont les plus sollicités pour assurer la formation nécessaire $(59,3 \%$ des PMI de l'industrie manufacturière). Le donneur d'ordres vient en deuxième position $(39,6 \%)$ et devance légèrement les organismes privés spécialisés $(38,5 \%)$ et plus nettement les syndicats ou groupement professionnels $(26,7 \%)$.

En ce qui concerne les organismes publics, toute une structure d'écoles spécialisées et de centres techniques est en place au niveau national et régional, fonctionnant parfois conjointement sur fonds publics et privés et proposant des cycles de formation courts ou longs selon les cas. Si certains sont plus précisément axés sur la formation initiale, ils restent ouverts à des salariés envoyés par leur entreprise et organisent d'ailleurs souvent des stages spécifiques. Nombre de ces organismes et écoles spécialisées étant subventionnés, le coût des stages y est faible afin de permettre un accès facile, notamment aux salariés des PMI.

Le principe de ces organismes est d'allier enseignement pratique (incluant l'apprentissage de savoir-faire manuels avant de passer sur équipements automatisés) et enseignement théorique. Dans le cas de cycles longs, il est parfois proposé d'effectuer la partie théorique par correspondance et de regrouper la pratique autour de quelques stages courts, pour répondre aux besoins des PMI qui ne peuvent se passer de leurs salariés pendant une longue période. Ces organismes, dont l'école supérieure pour les PME de la Japan Small Business Corporation, sont surtout utilisés pour la formation des techniciens/ingénieurs (16\% des PMI). La faiblesse du pourcentage d'entreprises qui envoient leurs salariés à l'école supérieure pour les $\operatorname{PME}(4,8 \%)$ est liée au haut niveau de connaissances qui est requis à l'entrée. 
Parallèlement à l'activité de ces différents organismes et aux stages qu'ils proposent, de nouvelles procédures apparaissent, notamment dans le cadre de la révision de la "loi sur la formation professionnelle nationale (national vocational training law)", devenue en 1985 "loi pour la promotion du développement des compétences professionnelles (law to promote vocational ability development)". Les aides publiques sont généralement instituées au Japon dans le cadre d'une loi nationale qui en fixe les objectifs. La mise en oeuvre de la loi est par contre confiée à l'échelon régional qui dispose d'une certaine souplesse dans la définition précise des actions à mener.

Un projet de nouveau système de formation professionnelle : le plan Icho de la préfecture de Kanagawa.

Le plan Icho peut servir d'illustration à cette nouvelle tendance. Il a pour but d'améliorer la formation individuelle, en développant les compétences et qualifications qui seront nécessaires au monde industriel des années 1990. Ses principes fondamentaux résident dans l'association de la formation de base et de la formation avancée dans un système intégré reposant non seulement sur le hardware mais aussi sur le software. Les concepts qui en découlent sont : la flexibilité, la spécialisation et la dispersion géographique des lieux de formation, un environnement formationnel innovant et une formation dispensée par des spécialistes dans les domaines de hautes technologies.

Dans le cadre de ce plan, les écoles de formation professionnelles de la préfecture sont en cours de réorganisation et de ré-équipement. Parmi les onze écoles de formation professionnelle, une école de branche, et l'institut de formation professionnelle de Kanagawa qui entrent dans le projet, cinq ont déjà mis en oeuvre les nouveaux programmes 
de formation, couvrant autant les champs de qualifications pratiques que d'instruction académique. Sur les vingt huit formations prévues, dix neuf étaient déjà en place fin 1987.

En liaison directe avec le plan Icho, diverses mesures ont été prises, au niveau préfectoral, afin de promouvoir et aider les programmes de formation du secteur privé.

- Le rôle central des "facilitateurs"

Ce sont des personnes qualifiées responsables de la planification et du développement de programmes de formation pour les salariés d'une entreprise ou d'une organisation. Leur existence a été institutionnalisée en 1985, par la révision de la loi nationale. Fin 1987, des "facilitateurs" étaient déjà appointés dans près de huit cents entreprises, et la progression devrait être rapide dans les prochaines années. La préfecture offre à ces "facilitateurs" un site où ils peuvent étudier et se rencontrer pour échanger leurs expériences. Elle leur fournit également les informations nécessaires pour mener à bien leurs objectifs.

- Les programmes privés aidés par le gouvernement préfectoral

Si le programme prévu entre dans les normes définies par le plan Icho, en termes de contenu, de moyens éducatifs et de qualification des instructeurs, il peut être reconnu par le gouvernement préfectoral en tant que "programme de formation professionnel autorisé". Fin 1987, trente et un programmes de ce type, menés indépendamment par des entreprises, et vingt deux programmes conjoints, placés sous la responsabilité d'organisation de PME, avaient d'ores et déjà été agréés. Dans le cadre de cet agrément, la préfecture apporte une aide financière pour le paiement des salaires des personnels en cours de formation, ainsi qu'une participation aux coûts de 
mise en oeuvre et de réalisation des programmes lorsque ceuxci sont menés par des organisations de PME. De plus, un système de subvention particulier a été ouvert en 1987 pour des programmes de développement des compétences émanant de PME qui souhaitent se préparer à une diversification vers des domaines d'activité nouveaux (The Kanagawa, oct. nov. 1987) ${ }^{20}$.

Les actions de formation entrant dans le cadre de procédures de ce type ne sont pas encore généralisables à l'ensemble des PMI, ni d'ailleurs à la totalité des régions. Même si l'on peut supposer que leur importance va croître dans les années à venir, il reste à peu près certain qu'elles ne sont pas de nature à remettre en cause la prépondérance de l'interne en matière de formation des hommes. Elles ont pour vocation de donner à l'entreprise les moyens de concevoir et réaliser une formation intégrée qui s'appuie sur des compétences extérieures, tout en mobilisant les savoir-faire internes. Elles s'adressent plus particulièrement, aujourd'hui du moins, à des PMI très innovantes ou à des entreprises ayant à faire face à un changement de grande ampleur. Il est par contre probable que la grande majorité des PMI se tourneront encore en premier lieu vers les mécanismes traditionnels pour acquérir les compétences liées aux nouvelles fonctions qui apparaissent, et notamment pour ce qui est des entreprises sous-traitantes, vers le donneur d'ordres.

\subsection{L'aspect formateur des relations donneurs d'ordres/sous-traitants}

Les relations très étroites qu'entretiennent les entreprises japonaises appartenant au même système productif permettent une circulation de l'information et une diffusion de la formation qui à leur tour renforcent les relations. Celles-ci sont de deux natures : formelles par les structures de "clubs" et réunions ad- 
hoc à périodicité régulière; et informelles par l'importante circulation des hommes à l'intérieur de l'ensemble du système productif considéré.

Les relations formelles

La plupart des grandes entreprises, notamment dans l'industrie automobile ou dans celle de l'électronique grand public, gèrent des "clubs" ou associations de fournisseurs dont l'organisation et le fonctionnement diffèrent selon les firmes, mais dont les principes sont les mêmes dans tous les cas.

Prenons le cas d'un constructeur automobile mineur dont le "club"regroupe environ 200 entreprises. Les activités sont divisées en deux groupes : un groupe d'enseignement et un groupe d'affaires générales:

- Le groupe d'enseignement est composé de plusieurs catégories thématiques : gestion comptable, gestion de la qualité, gestion des prix, gestion des ressources humaines, sécurité. La participation à ces actions est volontaire. Sur chacun des thèmes, des sous-groupes sont formés ( 4 à 5 selon les cas) par secteur d'activité : emboutissage, fonderie... Chaque sous groupe se réunit une fois par mois.

- Le groupe d'affaires générales fonctionne sur la base d'une réunion générale annuelle ayant pour but d'annoncer les stratégies et politiques du groupe sur l'année; et d'une réunion annuelle par groupe professionnel pour échanger les informations générales nécessaires. Les groupes professionnels sont : équipements-machines, carrosserie, moteurstransmissions. De plus, une fois par mois les responsables des entreprises concernées se réunissent avec le personnel de direction du donneur d'ordres sur les questions commerciales. 
En plus de ces actions par groupes, il existe également des relations formelles bilatérales, c'est-à-dire du "conseil" 21 par entreprise, touchant un nombre plus restreint de firmes. C'est notamment le cas de la recherche et développement qui pour des raisons de stratégies et de secret est rarement l'objet des réunions de groupe. En gardant le même exemple, voici les actions de conseil bilatéral formalisées:

- conseil de portée générale axé sur :

- planification de la gestion comptable

- gestion des directives

- production mensuelle (pertes, profits...)

. résultats de l'année au niveau de la production

Sur ces quatre points, il s'agit à ce stade de la gestion quotidienne et non plus comme dans la structure de groupe de principes généraux.

- gestion globale (assuré par le service commercial)

. Total Quality Control (TQC)

- conseil par qualification :

- gestion de la qualité

- gestion des prix

- gestion de production

- niveau de production

- gestion comptable.

Ce conseil bilatéral touche, dans le cas de ce constructeur automobile, une trentaine d'entreprises. Ce nombre peut paraître faible, mais il faut garder plusieurs choses en mémoire:

- toutes bénéficient de la structure par groupe du "club"; 
- les entreprises ne bénéficiant pas du conseil bilatéral institutionnalisé peuvent par contre profiter des relations informelles dont nous allons parler ci-après pour obtenir le même type d'informations/formation;

- les grands fournisseurs ont eux-mêmes un "club" dont le fonctionnement est calqué sur celui du constructeur au sommet et par le biais duquel ils peuvent retransmettre les connaissances acquises aux niveaux inférieurs.

Les relations informelles

Les relations entre donneurs d'ordres et sous-traitants ne se limitent pas à ces procédures institutionnalisées. Une description détaillée de toutes les actions qui contribuent à la formation des hommes chez les fournisseurs et sous-traitants est impossible tant les exemples sont nombreux. En règle générale, on peut dire qu'il y a un incessant va et vient entre les uns et les autres. Les échanges de ce type furent très fréquents lors de la mise en place des systèmes de type kanban, des cercles de qualité et aujourd'hui, pour l'assimilation de l'analyse de la valeur et la compréhension de la gestion globale.

Ainsi, le donneur d'ordres envoie chez son fournisseur les personnels compétents pour une durée déterminée, plus ou moins longue selon le problème à résoudre. Sur la base des enquêtes de terrain réalisées, on peut estimer que du personnel du donneur d'ordres passe de 4 à 10 jours par mois chez un sous-traitant donné. Il peut s'agir de l'introduction de la CAO, ou de la mise en oeuvre d'un nouveau système de gestion de production, par exemple. Les personnels concernés par ces mouvements ponctuels et répétés, bien que souvent de courte durée, sont tout autant les opérateurs que les chefs d'équipes ou ingénieurs. Selon les problèmes qui se posent, ce ne sont pas les mêmes personnes qui se déplacent. 
De même les salariés d'un sous-traitant peuvent passer plusieurs jours (ou plusieurs mois) chez le donneur d'ordres afin d'apprendre, par exemple, le fonctionnement d'une nouvelle machine, la fabrication de prototypes, la CAO... Dans certains cas, tous les jeunes hommes recrutés par le sous-traitant comme "réguliers" suivent des cours chez le donneur d'ordres dans le cadre de leur formation à l'entrée.

Dans d'autre cas de sous-traitants très intégrés, il arrive que les machines soient fabriquées ou achetées par le donneur d'ordres qui met en place son propre système de leasing pour rétrocéder l'équipement au sous-traitant ${ }^{22}$. Toute la formation nécessaire est alors dispensée par lui, avec plus ou moins de garantie (dans le contrat même) d'utilisation exclusive de l'équipement à son profit.

Lors de la sortie de nouveaux produits, il y a toujours appel de personnel du fournisseur/sous-traitant chez le donneur d'ordres afin de les informer et former par rapport aux besoins spécifiques de ce produit. La conception des nouvelles pièces peut même se faire en collaboration entre du personnel des deux parties, afin de garantir une bonne correspondance des pièces avec les besoins exprimés. Dans ce cas, le personnel du sous-traitant travaille chez le donneur d'ordres, jusqu'à ce que le prototype soit au point.

Le changement technique, qu'il se situe au niveau de la production ou de l'organisation, n'a pas provoqué de rupture significative dans les relations entre donneurs d'ordres et soustraitants en ce qui concerne leur effet formateur. Bien qu'il soit généralement admis par les grandes firmes que, la maîtrise technologique des PMI s'étant accrue, il est aujourd'hui moins besoin de les guider (celles qui en avaient le plus besoin ont d'ailleurs souvent été éliminées par les diverses phases de restructuration qui ont eu lieu), il se manifeste une tendance au 
renforcement de ces liens éducatifs face aux nouvelles contraintes. Certes, il y a déplacement des contenus des aspects traités, passant de plus en plus de problèmes technologiques à des problèmes organisationnels ou gestionnaires, confirmant l'amélioration de la maîtrise technologique des sous-traitants concernés. Le constructeur automobile pris comme exemple illustre bien cette tendance. Son objectif, lié à l'impact de la hausse du yen qui entraîne l'entreprise vers une situation déficitaire, est de renforcer la structure de "club" afin d'élever le niveau général du système productif qu'il dirige et accroître ainsi sa compétitivité dans le contexte monétaire actuel.

Ces quelques exemples suffisent à montrer à quel point la formation est continuellement présente dans les entreprises japonaises, même si elle passe par des mécanismes particuliers qui ne sont pas comptabilisés comme tels. En effet, si par exemple, on met bout à bout les heures passées par des personnels des donneurs d'ordres à quelque niveau que ce soit (car tout cela se passe en cascade), pour des actions participant à la formation et à l'élévation du niveau technique de l'ensemble du système productif, ou si l'on additionne le temps passé par les uns et les autres à mener à bien une équipe de projet, on s'aperçoit vite que les frais de formation annoncés ne reflètent en rien la réalité. Ce constat ne doit toutefois pas faire oublier qu'un réel problème se pose aux PMI à qui l'on demande (ou à qui l'on va demander) de maîtriser de plus en plus de fonctions : innovation, développement, conception, nouvelles technologies, qualité, réseaux de communication et même internationalisation. Une telle évolution ne pourra se faire largement qu'avec une véritable mise à niveau du tissu des PMI pour laquelle l'importance accordée à la formation dans les mentalités et dans le fonctionnement quotidien est certes un atout, mais un atout aujourd'hui insuffisant. 
Face au changement technique et en relation avec les contraintes nouvelles dues à la hausse du yen et à l'internationalisation croissante, les PMI sont confrontées à l'inadéquation entre les savoir-faire à disposition et les besoins de nouvelles compétences. Pour résoudre ce problème, deux voies s'ouvrent à elles : le recrutement d'une main-d'oeuvre plus adaptée, ou la formation des salariés.

En ce qui concerne le recrutement, les possibilités offertes aux PMI sont limitées du fait de leur position défavorisée sur le marché du travail et du coût des campagnes d'embauche. De plus, la concurrence des grandes entreprises sera encore vive dans les années à venir relativement à la main-d'oeuvre que les PMI souhaiteraient embaucher. Bien que la mobilité soit plus élevée dans les PMI que dans les GE, un renouvellement massif des compétences internes n'est guère envisageable par recrutement direct.

En matière de formation, divers mécanismes ont fait la preuve de leur efficacité lorsqu'il s'agit de transmettre des compétences existant dans l'entreprise ou dans le système productif. Ils restent les vecteurs principaux de la diffusion des nouvelles compétences, mais ils ne suffisent plus car il faut d'abord acquérir les compétences en question. De plus, la maind'oeuvre disponible doit être en mesure d'assimiler la formation ce qui revient au problème des caractéristiques des salariés des PMI. Tenter de combler l'ensemble des besoins de compétences nouvelles par simple renforcement des modalités traditionnelles de formation, c'est-à-dire essentiellement par des procédures internes, n'est pas plus réaliste.

En fait, ces deux voies ne sont pas antagonistes et il semble bien, au contraire, que les PMI n'aient d'autre choix véritable que de développer des stratégies s'appuyant à la fois sur l'une et sur l'autre. 
L'élaboration des plans d'embauche vise, d'abord, à pourvoir les fonctions clefs en les confiant à des jeunes de haut niveau (diplômés d'université). Il est donc probable que les PMI auront dans les années à venir à faire un effort, notamment en terme de salaire, pour s'assurer quelques éléments de ce type. Ceux-ci, permettant l'internalisation de certaines connaissances nouvelles, devraient servir de levier à un retour à la formation interne et à l'extension de formules qui y participent bien que ce ne soit pas leur finalité première (équipes de projet par exemple). Mais pour ce faire, les PMI ont également besoin, et c'est encore un des objectifs des plans d'embauche, de s'assurer petit à petit une main-d'oeuvre capable de suivre les évolutions. Bien qu'il soit toujours délicat de se projeter dans le futur, il semble que l'exclusion des travailleurs âgés (plus de 45 ans) soit appelée à se poursuivre; les PMI qui ont largement servi de refuge à cette catégorie de main-d'oeuvre se tournant de plus en plus, même en ce qui concerne les recrutements en cours de carrière, vers des personnes plus jeunes (niveau baccalauréat).

Mais ces deux actions ne peuvent être que progressives, ce qui signifie que pour l'instant, le blocage du point de départ de la chaîne de formation interne demeure une préoccupation qui contraint les entreprises à revoir leur politique de recours à la formation mais l'articulation entre les procédures internes, souvent exclusivement utilisées, et l'externe, encore trop peu pris en compte. Les nouvelles politiques, telles que le plan Icho, qui tentent de se situer à ce niveau, c'est-à-dire de mettre en cohérence un apport extérieur avec la dynamique de formation interne devraient voir leur impact s'accroître. Toutefois, un problème reste à résoudre pour les PMI : le coût de ces actions. Même si bien des organismes extérieurs spécialisés sont subventionnés et donc peu coûteux, même si diverses procédures aidées se mettent en place au niveau national comme au niveau régional, il est bien évident que la mise en 
oeuvre de véritables stratégies de formation associant l'extérieur va provoquer un accroissement des budgets de formation. Toutes les PMI ne sont pas en mesure de se lancer dans cette voie et il est probable que la capacité à acquérir les compétences nouvelles qui émergent actuellement sera un élément primordial dans le repositionnement hiérarchique des entreprises pour les années quatre vingt dix.

\section{NOTES}

1.Sur toutes ces questions, voir pour plus de détail : Yveline LECLER, les PMI sous-traitantes japonaises face aux enjeux des années quatrevingt, in Les PME dans l'économie japonaise : un facteur de dynamisme, dossier $\mathrm{n}^{\circ} 10$ de l'Institut des Sciences du Travail. Université Catholique de Louvain, mars 1988, pp. 11-31.

\section{Idem, note 1 .}

3.Il est toujours très délicat de parler des PMI en général, tant cette catégorie est un ensemble hétérogène. Une distinction entre différents types de PMI serait souhaitable (PMI sous-traitantes de spécialité, de capacité, PMI innovantes, PMI traditionnelles, familiales etc...), car il est bien évident que les modes de réponse des unes et des autres ne sont et ne peuvent etre équivalents. Elles ne sont d'ailleurs pas toutes confrontées aux mêmes problèmes ou concernées avec la même intensité par les diverses évolutions en cours. Toutefois, faute d'enquêtes et données statistiques permettant de saisir cette complexité, il sera ici question de la situation moyenne des PMI, sachant que toutes sont malgré tout traversées, à un degré plus ou moins grand et selon des formes propres, par l'une ou l'autre des contraintes actuelles.

4.Sur ce point et en relation avec l'évolution de la politique salariale, voir Yveline LECLER, Le méritocratisme pragmatique : vers une nouvelle politique salariale au Japon, a paraitre in Annales des Mines: Gérer et Comprendre, septembre 1989. 
5.Voir Yveline LECLER, Précarisation de la main-d'oeuvre et réouverture des écarts entre petites et grandes entreprises, in Economie et Politique du Japon Contemporain, $\mathrm{n}^{\circ} 10$, EHESS/CRJC et CNRS/CDSH, juin 1981, pp. 23-65.

6. Est dit "travailleur en cours de carrière" toute personne qui a déjà été employé dans une autre entreprise et qui en la quittant, quelles qu'en soient les raisons, est sorti du système d'emploi régulier.

7.Chusho kigyo cho éd. (Secrétariat d'Etat aux PME éd.) Chusho kigyo hakusho 1987 nenpan (Livre blanc sur les PME 1987), p. 106.

8. Fujin rodo no jitsujo 1988 (situation du travail féminin), p. 37 annexe statistique.

9. Chusko kigyo cho éd. (Secrétariat d'Etat aux PME éd.) Chusho kigyo hakusho 1987 nenpan (Livre blanc sur les PME 1987), p. 108.

10. Les travailleurs qui atteignent l'âge de la retraite peuvent éventuellement poursuivre leur activité, soit par prolongement d'activité, soit par réemploi. Le premier cas de figure est surtout fréquent dans les PMI alors qu'il est très rare dans les grandes entreprises qui, si besoin est, préfèrent largement le système de réemploi qui revient en quelque sorte à une nouvelle embauche dans des conditions différentes et hors système d'emploi dominant. Sur ce point voir Rodo daijin kanbo seisaku chosa bu éd. (Cabinet du Ministre du Travail, bureau d'enquête sur les politiques éd.) 2000 nen no rodo (le travail en l'an 2000), p. 24.

11. Bien que l'emploi à vie soit la référence, il ne concerne qu'une partie de la main-d'oeuvre, y compris dans les grandes entreprises qui ont également recours a la main-d'oeuvre secondaire sans laquelle le système, trop rigide, ne pourrait perdurer.

12. Rodosho seisaku chosabu éd., (Ministère du travail, bureau d'enquete sur les politiques éd.) koyo kanri no jittai (situation réelle de la gestion de l'emploi), 1986, pp. 22-23.

13. Rodosho seisaku chosabu éd., op.cit ., pp. 44-45. 
14. Rodosho seisakubu éd., op.cit ., pp. 37-41.

15. Au Japon, la fin de l'année scolaire est au printemps. C'est donc à ce moment, et a ce moment seulement, qu'ont lieu les recrutements de jeunes sortant de l'école. C'est ce qui est appelé campagne de printemps, tant les entreprises entrent effectivement en campagne pour s'approprier les bons éléments.

16. Les sources utilisées ne permettent malheureusement pas d'approfondir cette question qui d'ailleurs mériterait de faire l'objet d'une recherche particulière dépassant le cadre de cet article.

17. NIEVR éd. (National Institute of Employment and Vocational Training, koyo shokugyo sogo kenkujo), naikuerekutoronikusu ka to seisangijutsu, shokuba soshiki no henka ni kansuru kenkyuhokokusho (rapport sur les changements de la structure des ateliers et les techniques de production face à la micro-électronique), mars 1985.

18. Voir C. MERCIER, chapitre 1 in : W. Cavestro, Y. Lecler, C. Mercier, Automatisation et dynamique du travail dans l'industrie des pièces pour l'automobile, comparaison France-Japon, Rapport au MRT, mars 1988.

19. Cet investissement n'était que partiellement réalisé en 1987. Le motif invoqué est qu'il avait fallu entre temps construire une nouvelle usine du fait de l'importance accrue prise par la transformation de la matière plastique. Le projet tient parait-il toujours mais a été différé (enquête de terrain en 1983 et 1987).

20. Diverses autres actions de formation trouvent leur place dans le cadre de l'Association pour le Développement des Compétences Professionnelles, créée comme dans les autres préfectures, sous l'égide de la loi du même nom.

21. Le mot "conseil" est utilisé pour traduire le terme anglais de "guidance" qui n'a pas d'équivalent strict en français.

22. Sur ce point voir C. Afriat et Y. Lecler. L'automatisation des PMI japonaises et son financement, in CPE Etude, $\mathrm{n}^{\circ} 70$, juin 1986. 


\section{SUMMARY}

Faced with technological change in relation to new constraints imposed by increasing internationalisation and the rise of the yen, small and medium-sized enterprises are finding themselves up against the inadequacy of the know-how at their disposal, and the need for new skills. There are two avenues offering a solution to this problem, namely staff training and the recruitment of a more suitable workforce.

In fact, these two possibilities are not inamical. Indeed, it seems that the SME have no real choice other than to develop strategies based on both.

As for the unions, they are generally positive and support whatever steps are taken. It is also the moment to consider quality circles outside their purely Japanese cultural context. They are certainly not unique to Japan, and can be brought to bear just as effectively in other contexts. 